OPEN

SUBJECT AREAS:

SOLAR CELLS

QUANTUM DOTS

Received

15 July 2013

Accepted

29 August 2013

Published

19 September 2013

Correspondence and requests for materials should be addressed to J.K.K. (jeung@kaist.ac.

* These authors contributed equally to this work.

\section{Broadband energy transfer to sensitizing dyes by mobile quantum dot mediators in solar cells}

\author{
Gede Widia Pratama Adhyaksa*, Ga In Lee*, Se-Woong Baek, Jung-Yong Lee \& Jeung Ku Kang
}

Graduate School of EEWS (WCU), Department of Materials Science and Engineering, KAIST Institute for the NanoCentury, Korea Advanced Institute of Science and Technology (KAIST), 291 Daehak-ro, Yuseong-gu, Daejeon 305-701, Republic of Korea.

The efficiency of solar cells depends on absorption intensity of the photon collectors. Herein, mobile quantum dots (QDs) functionalized with thiol ligands in electrolyte are utilized into dye-sensitized solar cells. The QDs serve as mediators to receive and re-transmit energy to sensitized dyes, thus amplifying photon collection of sensitizing dyes in the visible range and enabling up-conversion of low-energy photons to higher-energy photons for dye absorption. The cell efficiency is boosted by dispersing QDs in electrolyte, thereby obviating the need for light scattering ${ }^{1}$ or plasmonic ${ }^{2}$ structures. Furthermore, optical spectroscopy and external quantum efficiency data reveal that resonance energy transfer due to the overlap between QD emission and dye absorption spectra becomes dominant when the QD bandgap is higher than the first excitonic peak of the dye, while co-sensitization resulting in a fast reduction of oxidized dyes is pronounced in the case of lower QD band gaps.

r he most critical challenge in solar cell systems is to harvest photons in the visible and infrared (IR) regions. A class of ruthenium-complex dyes absorbs in the visible region and has been used extensively as photon collectors in dye-sensitized solar cells (DSSCs). However, the photon-to-current efficiency depends on the photon absorption intensity of the dye, which requires improvement. Thus far, the intensity has been improved by adding light scattering ${ }^{1}$ or embedding plasmonic ${ }^{2}$ structures. These approaches make such solar cells thicker than preferable, thus preventing the realization of thin transparent solar cells and leading to high resistances.

Photon co-absorbers ${ }^{3}$ attached onto a $\mathrm{TiO}_{2}$ surface can extend the absorption range to the visible region, thus resulting in improved photon collection. However, this approach requires a strict bandgap alignment of the coabsorbers with that of the $\mathrm{TiO}_{2}$ along with the potential energy states of the electrolyte and is limited by the poor contact of the co-absorbers to the $\mathrm{TiO}_{2}$ surface. Enhanced photon absorption intensity and efficiency in DSSCs have also been achieved by dissolving an energy relay dye ${ }^{4}$ in DSSCs. Meanwhile, this system is also limited to harvest a broad region of visible light.

Herein, we report that mobile quantum dots (QDs) can serve as mediators to receive and re-transmit broadband energy to sensitized dyes, thereby amplifying the photon collection intensity of sensitizing dyes in the visible range (300-800 $\mathrm{nm})$ and broadening the overall spectra up to the near infrared range $(900-1200 \mathrm{~nm})$ via the two-photon collection process. This phenomenon boosts the efficiency of cells by more than $200 \%$ exceeding that obtained with scattering and plasmonic structures on a similar framework. Therefore, these QDs provide an improved efficiency of DSSCs via amplified broadband photon collection at the visible and the near-IR ranges, which heretofore have remained unexploited in solar cells. The superposition of dyes and QDs can be distinguished by three different mechanisms: Föster resonance energy transfer (FRET) ${ }^{4}$ (Figure 1a), co-sensitization enabling the efficient reduction of an oxidized dye (Figure 1b), and two-photon absorption (TPA) allowing for up-conversion of low-energy photons to higher-energy photons for dye absorption (Figure 1c). The QDs were dispersed in an electrolyte, while the dyes were attached to the $\mathrm{TiO}_{2}$. The absorption and efficiency of the cells are doubled by dispersing QDs in the ionic liquid electrolyte of the DSSCs. We provide two sets of data supporting the role of QDs: 1) optical spectroscopy data showing that QDs serve as effective photon mediators once they are placed in the electrolyte, significantly increasing the broad absorption of the sensitizing dye throughout the entire range of the visible spectrum, and 2) external quantum efficiency (EQE) measurements showing that QDs markedly improve the photon-to-current efficiency via broadband absorption. 
a

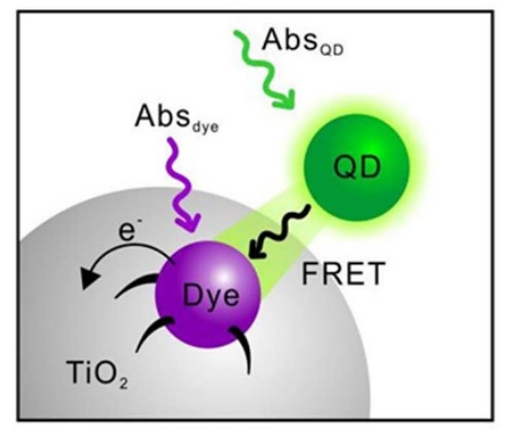

b
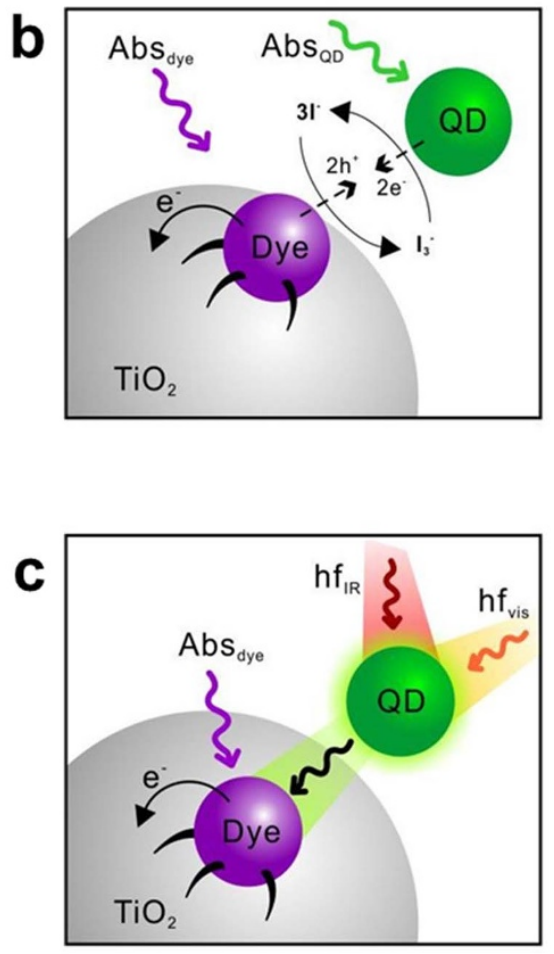
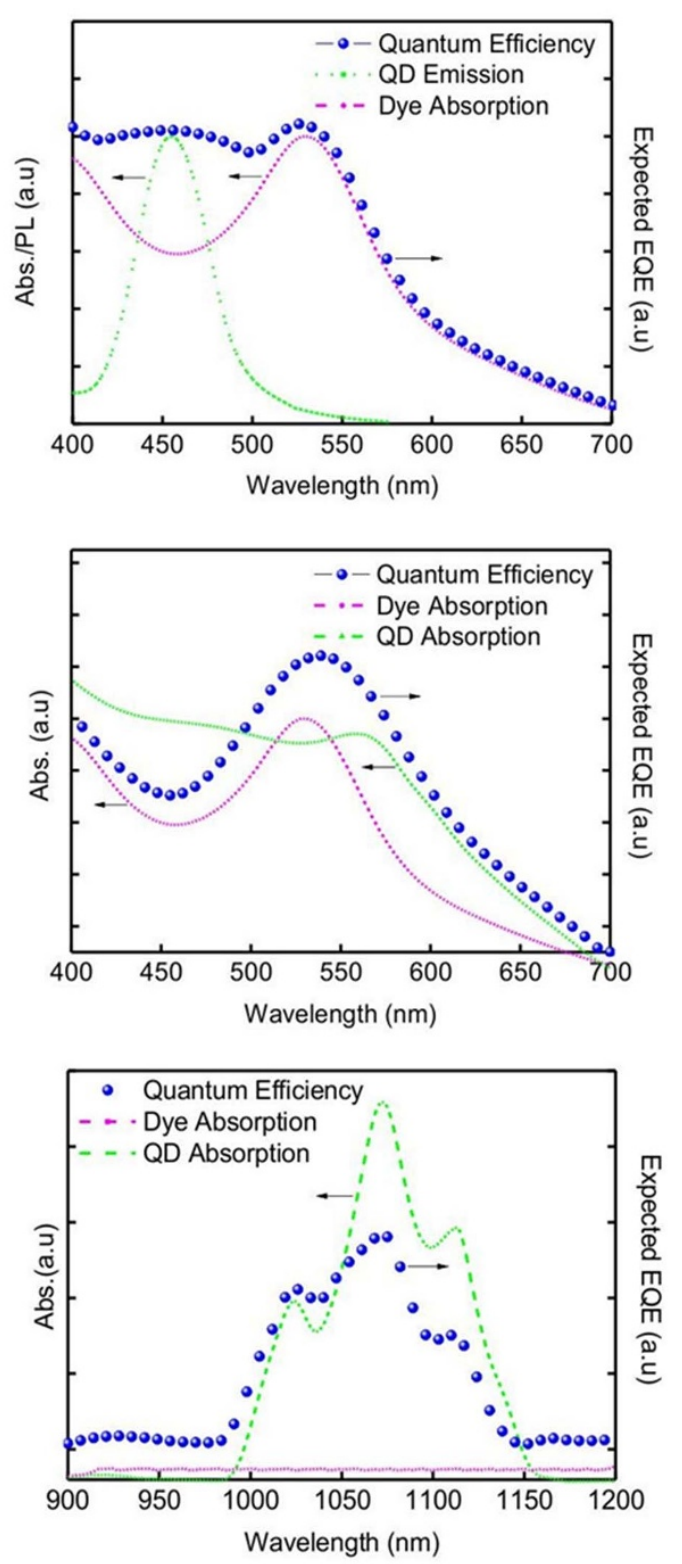

Figure $1 \mid$ Proposed mechanism (left) and expected spectra (right) for broadband energy transfer via. (a) Föster resonance energy transfer (FRET), where the QDs transfer the energy to the dye due to a strong resonant frequency (indicated by the overlap between the absorption of the dye and the emission of the QD). (b) Co-sensitization, where the QD helps the dye co-absorb photons in a different range and then activates the redox regeneration, thus accelerating the generated electrons to the $\mathrm{TiO}_{2}$. (c) Two-photon absorption, where the QD is capable of simultaneously absorbing high (Vis-range) and low (IR-range) photon energies, thus inducing energy transfer to the dye via resonance. These contributions increase and broaden the overall external quantum efficiency (EQE).

\section{Results}

Functionalized quantum dots in ionic liquid electrolyte. Prior to the assembly of the DSSC construct, we carried out a series of experiments to obtain the QDs with the appropriate functionality $y^{5-7}$ and to verify their energy transfer properties in the presence of the components that would ultimately be introduced into the DSSCs. CdSe QDs with mixed amino ligands (hexadecylaminetrioctylphosphineoxide-trioctylphosphine) were synthesized with four different bandgaps $(2.64 \mathrm{eV}$, green; $2.44 \mathrm{eV}$, yellow; $2.25 \mathrm{eV}$, orange; $2.21 \mathrm{eV}$, red). These ligands were exchanged with a thiol ligand (2-(dimethyl amino) ethane-thiol) and were dispersed in a conductive ionic liquid (1-ethyl-3-methylimidazolium bis (trifluromethylsulfonyl) imide) using the extraction process within solution phases as illustrated in the Supplementary Information (Figure S1a). The electrolyte was mixed with a proper combination of cation (EMIm-I or DMPII), anion $\left(\mathrm{I}_{2}\right)$, charge transport enhancer
(LiI), recombination inhibitor (tBP), and water ${ }^{8}$. Then, the solution was directly injected into the DSSCs (Figure S1b). Absorption (Figure S2) and emission (Figure S3) spectra were measured for these four different QDs and each spectrum was compared to that of the corresponding original amino-functionalized QD. The absorption and emission spectra of all of the QDs were basically maintained in the ionic liquid with slightly blue shifts. These blue shifts were attributed to the higher confinement energy of the QDs in the ionic liquid systems ${ }^{19-21}$.

Self-arrangement of quantum dots in DSSCs. There are two states in which QDs reside inside the DSSCs; first, they are mobile in the electrolyte, and second, they penetrate into the pores of the photoelectrode. To determine how the QDs are arranged in the solar cells, we performed an ex-situ analysis on two domains: 1) the electrolyte, and 2) the $\mathrm{TiO}_{2}$ photo-electrode. First, we imaged the ionic liquid 
containing the CdSe QDs using transmission electron microscopy (TEM). According to the TEM images, the QDs are homogeneous in size and are well dispersed without agglomeration in the electrolyte (Figure S4a-d). Second, we acquired a cross sectional image of the $\mathrm{TiO}_{2}$ photo-electrode using scanning electron microscopy (SEM). According to SEM elemental mapping based on energy dispersive $\mathrm{X}$ ray spectroscopy (EDX), the CdSe QDs permeate into the $\mathrm{TiO}_{2}$ photo-electrode (Figure $\mathrm{S} 4 \mathrm{e}-\mathrm{f}$ ). This result may be due to the smaller sizes of the QDs $(2.16-3.60 \mathrm{~nm})$ relative to the pores of the $\mathrm{TiO}_{2}(\sim 18.2 \mathrm{~nm})$. Although most of the QDs are present in the electrolyte as expected, it is inevitable that some of the QDs will attach to the surfaces of the $\mathrm{TiO}_{2}$ nanoparticles (NPs). To quantify the amount of QDs attached to the $\mathrm{TiO}_{2}$ surfaces, we provide quantification based on UV-Vis spectroscopy analysis (Figure S5). The analysis shows that the amount of QDs present on the $\mathrm{TiO}_{2}$ is much lower than that available in the electrolyte, thus indicating that the QDs are mobile. In fact, the synergy of these two domains was directly examined from their photovoltaic characteristics.

Broadband enhancement by QDs and their solar cell characteristics. An ionic liquid mixture of CdSe QDs and redox compounds was introduced into the DSSCs to evaluate their effect on performance. The devices containing green QDs, yellow QDs, orange QDs, and red QDs, are herein denoted as G-QD, Y-QD, O-QD, and $\mathrm{R}-\mathrm{QD}$, respectively. The absorption of the bulk device shows significant enhancement upon the introduction of QDs compared to the reference cells. The maximum value is observed for $\mathrm{R}-\mathrm{QD}$, with progressively lower values for O-QD, Y-QD and G-QD (Figure 2a). This trend is confirmed by EQE measurements, where the photonto-current efficiency is on the highest for R-QD, and decreases for $\mathrm{O}-\mathrm{QD}, \mathrm{Y}-\mathrm{QD}$, and G-QD (Figure 2b). This trend indicates that the dye absorbs additional energy supplied by the QD mediators. The EQE results were confirmed by the $J-V$ curves, which exhibit the same trend of enhancement (Figure 2c). The power conversion efficiency (PCE) obtained when using QD mediators is $4.62 \%$ for RQD, 4.44\% for O-QD, 3.56\% for Y-QD, and 3.26\% for G-QD compared to a PCE of $2.31 \%$ for the reference cells (Supplementary Information Table S1). All of the QDs, regardless of their size, successfully improve the photon absorption of the dye, thereby increasing the efficiency of the cells throughout the entire UVvisible range (300 to $800 \mathrm{~nm}$ ).

The EQE enhancement obtained for the DSSCs containing QDs reveals their exceptional performance compared to DSSCs containing plasmonic and scattering nanoparticles (NPs) (Figure 2d). The plasmonic and scattering layers were constructed by embedding $10 \mathrm{~nm}$-sized silver NPs and $400 \mathrm{~nm}$-sized titanium dioxide NPs, respectively. The enhancement due to the plasmonic NPs was similar to that of scattering NPs in the range of 300-450 nm and 650$750 \mathrm{~nm}$, in which the plasmonic particles exhibited an approximately 2 fold higher EQE. This trend may be due to the smaller sizes a
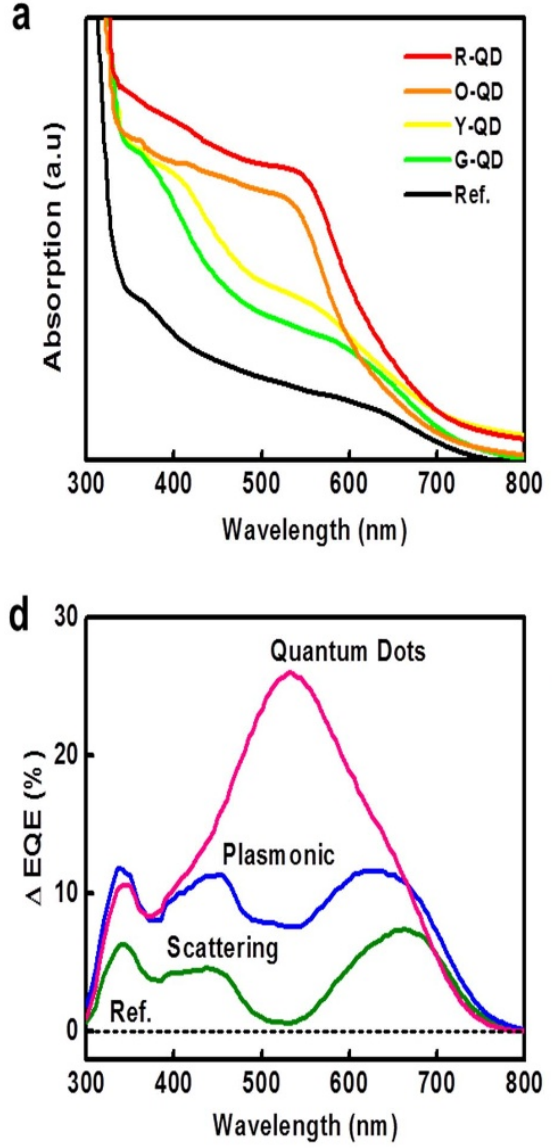

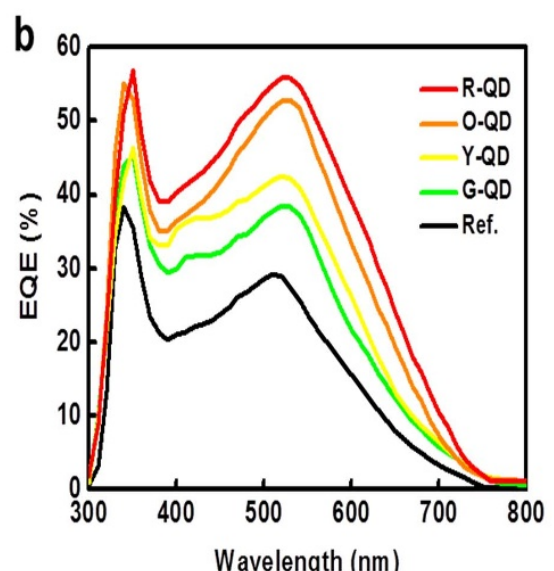

e

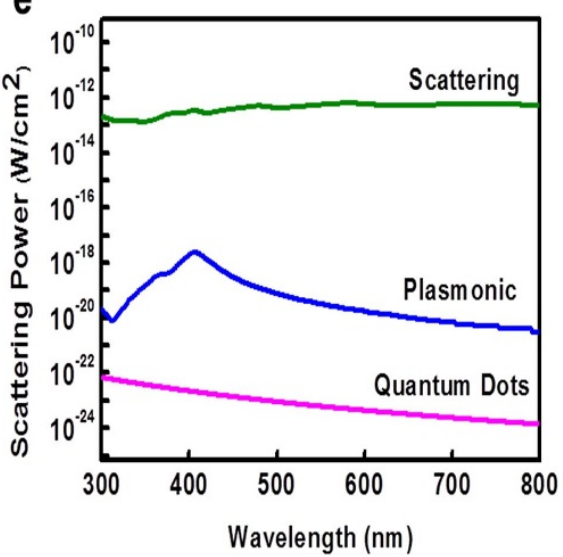

C

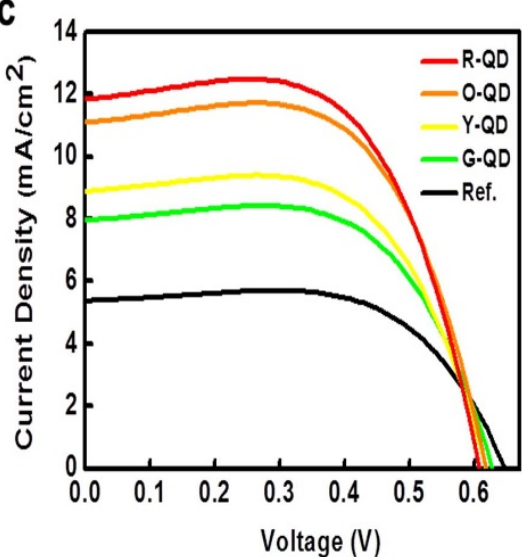

$f$

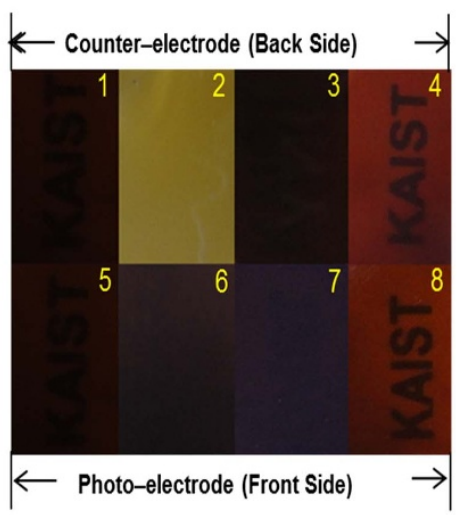

Figure $2 \mid$ Broadband enhancement by QDs and their solar cell characteristics. (a) Absorption of bulk solar cells containing different CdSe quantum dots (QDs). (b) External quantum efficiency (EQE) of solar cells with QDs showing broadband energy transfer. (c) Current density-voltage ( $J-V$ ) curve of solar cells under the AM 1.5 standard; the solar cell parameters are tabulated in Table S1. (d) EQE enhancement of solar cells containing QDs (red QDs; pink line), Ag plasmonic nanoparticles ( $\sim 10 \mathrm{~nm}$; blue line), $\mathrm{TiO}_{2}$ scattering layer ( $\sim 400 \mathrm{~nm}$; green line), and reference cells (without QDs; black dashed line). (e) Calculated scattering power of individual CdSe QDs (pink line), Ag plasmonic (blue), and $\mathrm{TiO}_{2}$ scattering layer (green). (f) Images of electrodes on the reference devices $(1,5), \mathrm{TiO}_{2}$ scattering layer $(2,6), \mathrm{Ag}$ plasmonic nanoparticles $(3,7)$, and CdSe QDs $(4,8)$. The solar cells employing QDs exhibit semi-transparency on both sides (photo and counter-electrode). 
of the plasmonic NPs in comparison to scattering NPs, which allow them to permeate into the porous photo-electrode and interact closely with the dyes. The unusual enhancement observed for the QD mediator far exceeds that of the cells containing scattering and plasmonic NPs, particularly in the range for the first excitonic peak of the dye (450-650 nm). The cross-section of scattering power for the QDs, plasmonic NPs and scattering NPs is also calculated via the optical simulation (Figure 2e). More details for the optical simulation have been described in the Supplementary Information. The scattering NPs exhibit the highest power over the plasmonic NPs and the QDs in the entire visible range (300-800 $\mathrm{nm}$ ). This indicates that the EQE enhancement by scattering NPs is originated mainly from the optical photon scattering. Meanwhile, the plasmonic NPs can localize the electric field of photons, as supported by the appearance of the peak in the range of $380-480 \mathrm{~nm}$. This localized field can amplify the photon absorption of the dye; therefore it has a similar enhancement to that by scattering NPs, albeit with an approximately double EQE enhancement. However, the EQE enhancement by the QDs cannot be explained in terms of a localized surface plasmon because the free electron density of the QDs is not significant enough to provide a resonance interaction with the incoming photons unless doping atoms are introduced into the $\mathrm{QDs}{ }^{10}$. Moreover, the phenomenon cannot be attributed to increase optical scattering of photons because the QDs is much smaller than the wavelength of visible light. This deduction implies that the QDs most likely through a relatively different mechanism.

An additional feature of the DSSCs containing QDs is their employment in semi-transparency devices, compared to the reference and those with plasmonic or scattering NPs (Figure 2f). Qualitatively, the transparency of the thin cells with the QDs is comparable on both sides: the photo-electrode and the counterelectrode. The QDs is quite stable in the ionic liquid. When light passes through the electrolyte, the QDs filter the light through photon absorption, thus reducing the probability of direct photon absorption by the redox ions. Consequently, upon light absorption by the QDs, radiative decay will occur by photon emission, which may explain why the cell containing QDs in ionic liquid electrolyte exhibits a higher transparency (Figure S6) compared to the cell without QDs. In addition, the electrolyte containing QDs penetrates to the porous photo-electrode, thus making both sides of devices semitransparent. This result enables DSSCs to receive more photons from both sides ${ }^{11}$, which leads to an increased efficiency. In contrast to the normal belief that a darker cell has a higher absorbance capacity, we find that mobile QD-mediated solar cells are exceptional and may be used in the fabrication of thin high-efficiency solar cells.

We tried to correlate the EQE (Figure 2b) based on the optical feature of absorption (Figure S2) and emission (Figure S3) of the QDs. The enhancement over the full range spectra can be explained by the broadband enhancement via two different linear energy transfer mechanisms: Föster resonance energy transfer and Co-sensitization enabling the efficient reduction of oxidized dyes, as well as a non-linear energy transfer process involving two-photon absorption. All QDs used in this study show different sequential enhancement in different mechanism which is more correlated with their bandgap relative to the dye.

Föster resonance energy transfer (FRET). The contribution of the FRET effect can be clearly observed by employing a thin layer of photo-electrode $(\sim 4 \mu \mathrm{m})$ in the DSSCs. We found that all of QD devices exhibited a higher EQE through the entire visible range compared to the cell without QDs (Figure S7). The cell with GQD showed the highest EQE values, followed by $\mathrm{Y}-\mathrm{QD}, \mathrm{O}-\mathrm{QD}$, and R-QD. The maximum EQEs of devices employing QDs correspond to approximately $530 \mathrm{~nm}$, which closely corresponds to the first excitonic absorption of the dye at $533 \mathrm{~nm}$. This indicates that the QDs direct additional photons to the dye, therefore improving the dye absorption. To investigate how the QDs improve the dye absorption, we normalized all EQEs according to their wavelength at the maximum EQE (Figure 3a). We found "bump" EQEs for GQD and Y-QD, in contrast to "steep" EQEs for O-QD and R-QD at similar ranges of $380-530 \mathrm{~nm}$. The reference EQE is positioned lower in than the "bumps", but higher than the "steep" EQEs. We also utilized a hybrid QD by mixing G-QD and Y-QD in a device construct, which displays an even higher EQE compared to the single-QD devices (G-QD or Y-QD), as shown by Figure $3 \mathrm{~b}$.

Optical spectroscopy measurements were conducted to explain the EQE (see method). Figure 3c shows the emission-absorption overlap between the QDs and the dye. The broad absorption spectrum of the dye (400-700 nm) shows good overlap with the emission of all of QDs (430-679 $\mathrm{nm}$ ). This overlap is a fundamental requirement for energy transfer (ET) between the QDs and the dye, which is driven by their molecular transition at the resonance frequency ${ }^{4,9}$. The G-QD and Y-QD have the emission peak at $488 \mathrm{~nm}(2.54 \mathrm{eV})$ and $521 \mathrm{~nm}(2.38 \mathrm{eV})$, respectively. These QDs are more likely to transfer energy to the dye (first excitonic peak at $533 \mathrm{~nm} ; 2.32 \mathrm{eV}$ ) than O-QD (564 nm, $2.12 \mathrm{eV}$ ) and R-QD (599 nm, $2.07 \mathrm{eV})$. The ET preferably occurs from higher emission energies (2.54 eV for G$\mathrm{QD}$, and $2.38 \mathrm{eV}$ for $\mathrm{Y}-\mathrm{QD})$ to lower absorption energies $(2.32 \mathrm{eV}$ for the dye). The efficiency of the ET was calculated based on emission time decay measurements (Figure S8). First, the dye was gradually added in various concentrations to G-QD in a solution mixture. The emission lifetime $(\tau)$ of G-QD decreased gradually with increased dye concentrations (Figure 3d), indicating a successful ET from G-QD to the dye. Second, in a similar way, Y-QD was added to G-QD, and it was revealed that ET occurred from G-QD to $\mathrm{Y}-\mathrm{QD}$, albeit with less efficiency (Figure 3e). Third, redox ions were added to the solution mixture of the G-QD (Figure 3f). ET still took place, but it showed a photo-bleaching effect of the QDs by the redox ions ${ }^{4-7}$. The ET was estimated by adopting the FRET theory reported by Lunz et al. ${ }^{9}$ using the following equation:

$$
\mathrm{ET}=\frac{1}{1+\frac{2 R^{4}}{C_{a c c} \cdot \pi \cdot R_{0}^{6}}}
$$

where $\mathrm{R}$ is the distance between the QDs (transmitter) and the acceptor species, $C_{\text {acc }}$ is the acceptor concentration, and $R_{0}$ is the Föster radius. We estimated the ET for the aforementioned sets at approximately $40.9 \%$ from G-QD to the dye and $12.9 \%$ from G-QD to $\mathrm{Y}-\mathrm{QD}$. The presence of redox compounds could be detrimental for the ET with its deficiency of $7.6 \%$.

According to these optical spectroscopy analyses, the EQE results indicate that the EQE enhancement caused by the QDs could be driven by a strong emission-absorption overlap between the QDs and the dye, which leads to ET. However, the more dominant ET is driven by G-QD and Y-QD rather due to their higher emission energy in comparison to the absorption energy of the dye. An even higher ET could be achieved by mixing G-QD and Y-QD, so that the ET would occur through a cascade process from G-QD to Y-QD and then from Y-QD to the dye (in addition to the direct ET from G-QD to the dye). This can be proven by the highest EQE obtained using the hybrid QDs. The EQE enhancement in the range of 430-533 nm could be explained by the contribution of emission spectra from G-QD and Y-QD. However, the EQE enhancement appears to be started from $380 \mathrm{~nm}$ and this blue shift might be due to the higher reflective index medium of QDs inside the real device compared to the solution mixture. According to the FRET mechanism, at the first excitonic peak of the dye $(533 \mathrm{~nm})$ that the maximum for emission of QDs could contribute for ET, the G-QD and Y-QD have "bumps", but O-QD and R-QD have "steeps". The EQE data also shows significant enhancement in the range of 533-800 nm, which implies that the FRET is not the single effect, instead there must be another mechanism governing the latter enhancement range. 
a

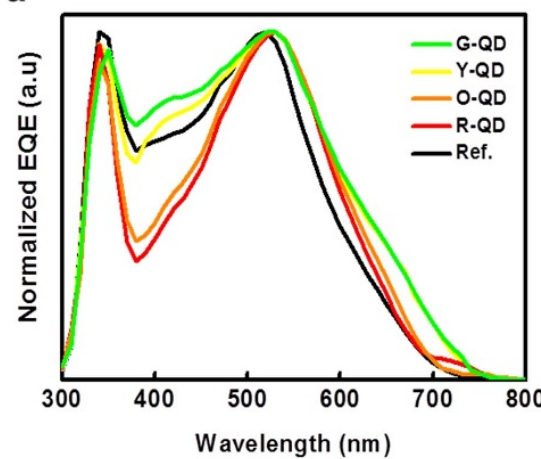

d

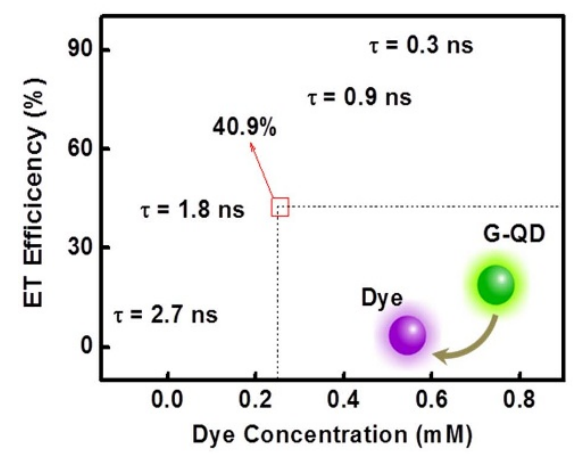

b

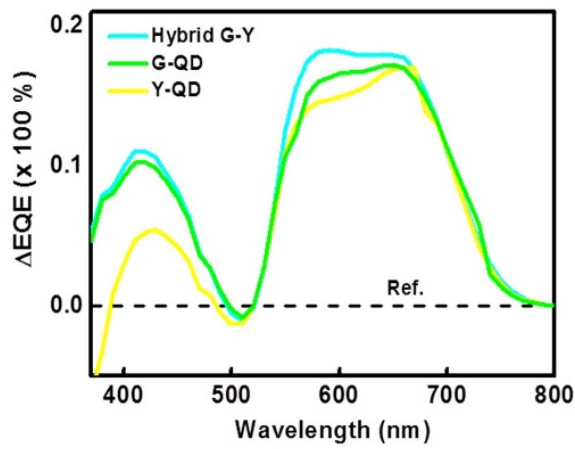

e

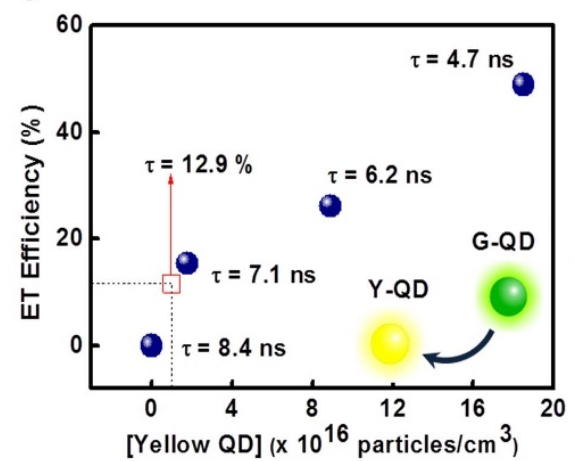

C

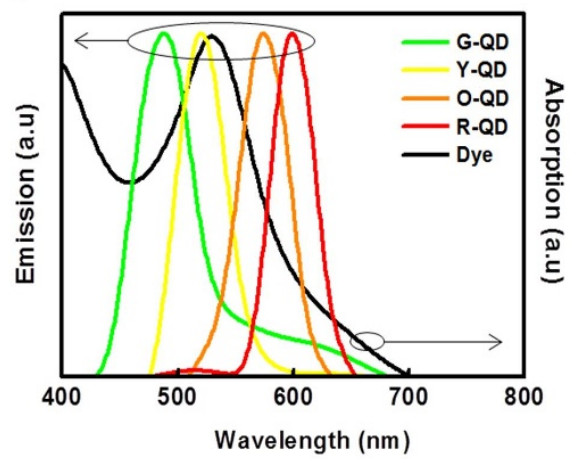

f

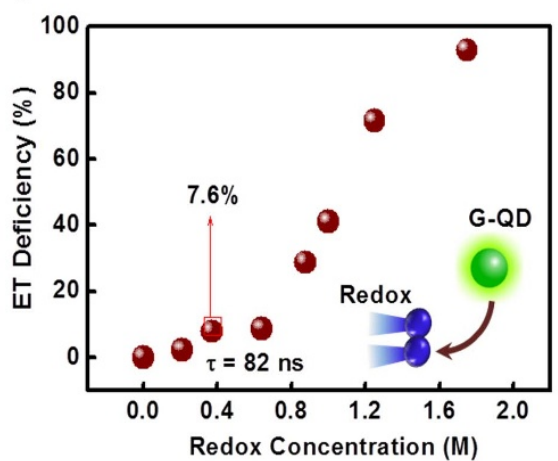

Figure $3 \mid$ Föster resonance energy transfer (FRET). (a) Normalized EQE of the solar cells (the EQEs were normalized to more clearly demonstrate the FRET effect). (b) EQE enhancement of hybrid QDs (mixed G-Y QDs). (c) Absorption-emission overlap between N719 dye and CdSe QDs. Energy transfer (ET) efficiency versus concentration between: (d) Transmitter G and receiver dye; (e) Transmitter G and receiver Y; (d) Deficiency of ET due to photo-bleaching of transmitter $\mathrm{G}$ to redox ions.

Co-sensitization enabling the efficient reduction of oxidized dyes. To analyze this effect, we examined the device performance with a thicker photo-electrode $(\sim 10 \mu \mathrm{m})$. First, we measure the back side EQE (Figure 4a). Directing the photon toward the back side electrode of the solar cells enables us to collect a higher spectra response generated from the electrolyte containing QDs. The results showed that all QDs give a higher EQE response compared to the reference cell. R-QD gives the highest EQE, followed by $\mathrm{O}-\mathrm{QD}, \mathrm{Y}-\mathrm{QD}$, and G-QD. Accordingly, a similar trend was also observed for the front side EQE, as shown in Figure S9. The enhancement occurs is in the range of 500 to $800 \mathrm{~nm}$ with a slight red-shift as the EQE increases. It should be noted that the QDs do not absorb photons in the range of 620-800 nm. Typically, the absorption loss of DSSCs is partly due to photon absorption by redox ions in the electrolyte, which does not contribute to the current generation. The presence of QDs in the electrolyte reduces the absorption loss due to the photon filtering process of the QDs in the electrolyte. Figure 4a supports that the presence of the QDs in an electrolyte plays their role in reducing this loss. Consequently, these results imply that the QDs most likely mediate the reduction process between the dye and the redox ions.

To justify the above hypothesis, we plotted the dynamic trend of the redox diffusion coefficient versus light intensity (Figure 4b), which were derived from electrochemical impedance spectroscopy (Figure S10). All of the QDs accelerate redox diffusion in the electrolyte, which is desirable for achieving high-efficiency devices. The diffusion enhancement trend is matched by the EQE data. The process is more efficient at higher photon illuminations, which implies that the presence of the QDs alone will not affect the redox diffusion if not enough light is present. Meanwhile, the statistical data of the back side EQE demonstrate that the QDs do not significantly affect the electro-catalytic characteristics of the platinum counter electrode (Figure S11).
To further examine the enhancement trend, we provide absorption data for all of the QDs and dye in solution (Figure 4c). There is a strong absorption overlap between the dye and the QDs; R-QD has the greatest overlap with the dye, followed by O-QD, Y-QD, and GQD. The characteristics of the absorption overlap are related to the co-sensitization concept. However the QDs do not directly co-sensitize the $\mathrm{TiO}_{2}$ due to their mobile state in the electrolyte. In addition, the absorption transient of the dye attached to the $\mathrm{TiO}_{2}$ was examined in the presence of redox ions and redox-containing QDs. The absorption transient (see Methods) is assigned to the oxidized state of the N-719 dye used in this study. We estimated the lifetime of oxidized dye for each curve: $30 \mu$ s for R-QD, $38 \mu$ s for O-QD, $44 \mu$ s for $\mathrm{Y}-\mathrm{QD}, 50 \mu \mathrm{s}$ for $\mathrm{G}-\mathrm{QD}, 58 \mu$ s for redox only, and $163 \mu$ s for no redox (see Methods). The lifetime indicates how quickly the oxidized dye can be regenerated by redox ions in an electrolyte. This examination reveals that $\mathrm{R}-\mathrm{QD}$ is most efficient for co-sensitization enabling the fast regeneration of the oxidized dye, followed by $\mathrm{O}-$ QD, Y-QD, and G-QD. The redox reduces the absorption lifetime of the oxidized dye, which is further decreased by the addition of QDs (showing the same trend).

Because the QDs do not absorb in the range of 620-800 nm, the EQE enhancement in this range does not originate from increased photon absorption, but from the mediation of QDs in activating the reduction process between the dye and the redox ions. The driving force for this trend is the absorption overlap between the QDs and the dye. This overlap is most likely due to co-photon absorption channels: one channel for direct electron injection to the $\mathrm{TiO}_{2}$ and another channel to activate the redox reduction toward the dye. The nature of the thiol surface ligands on the QDs may also play a role in this phenomenon ${ }^{12,13}$.

Two-photon absorption (TPA) and its photon-to-current efficiency. The superposition of the dye-QDs in our system enables the 
a

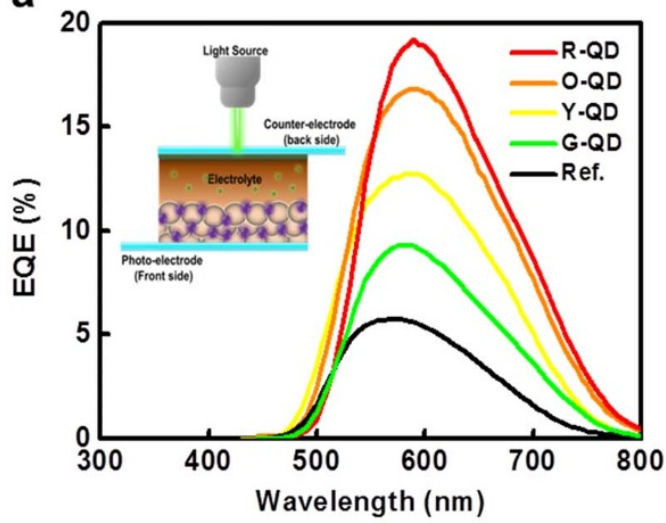

C

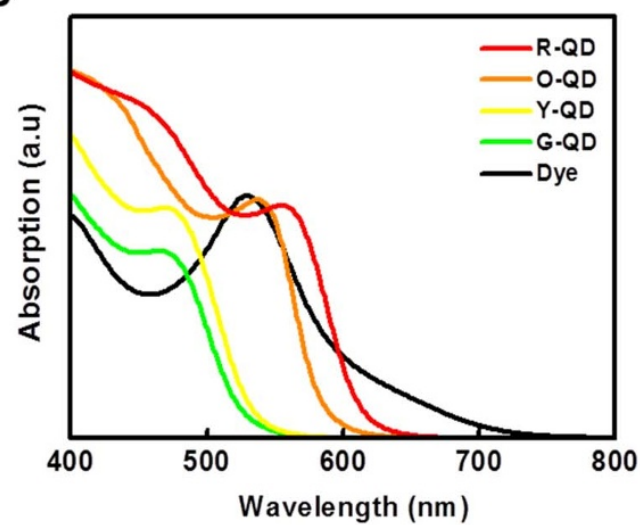

b

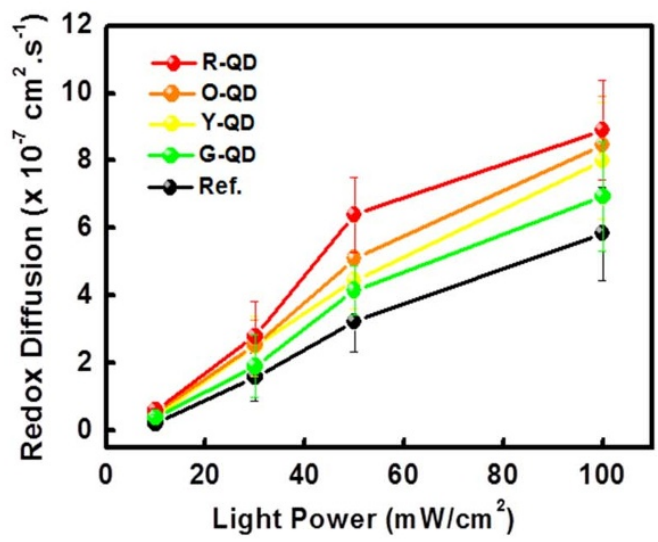

d

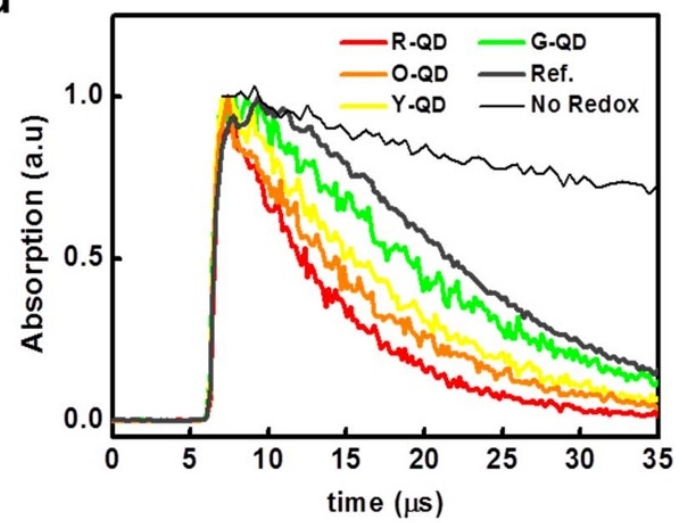

Figure $4 \mid$ Co-sensitization enabling the efficient reduction of oxidized dyes. (a) Back side EQE of solar cells (inset: illustration of a photon directed to the counter electrode side to collect higher EQE responses generated by the electrolyte region). (b) Dynamics of redox diffusion $\left(D_{\text {red }}\right)$ versus illumination power ( $D_{\text {red }}$ was calculated from electrochemical impedance spectroscopy). (c) Absorption-absorption overlap between N719 dye and CdSe QDs.

(d) Absorption time decay of oxidized dye attached to $\mathrm{TiO}_{2}$ films measured in a solution containing redox components and QDs.

demonstration of two-photon absorption (TPA) ${ }^{14}$. The TPA process was confirmed by absorption spectroscopy and EQE measurements in the near IR range (900-1200 nm). We compared the primary (300-600 nm) and secondary (900-1200 nm) absorption of CdSe QDs capped with original amino-based ligands in toluene, where the secondary absorption occurred in the near IR range (Figure 5a). These secondary absorption energies are approximately half of the primary absorption energies in the visible range and with absorption intensities $42 \%$ lower than the primary intensities.

More pronounced confined peaks for secondary absorption were observed after the thiol functionalization of CdSe QDs in ionic liquid solution (Figure 5b). A red shift was observed as the QD size increased. This behaviour implies that the thiol ligands covering the QD surfaces and the high cation-anion density of the ionic liquid surrounding the QDs may play a role in redistributing the electronic charges, thus increasing the confinement of energy states. Regarding this signature, an interesting question arises as to whether the secondary absorption at the lower photon energies contributes to the photon-to-current generation.

To assess the improvement of the photon-to-current efficiency within the IR range, EQE measurements were performed (Figure $5 \mathrm{c}$ ). The near-IR EQEs (900-1200 nm) observed for the cells with QDs are typically small (see Methods) compared to their visible EQE (300-800 nm). The yellow QDs exhibited the highest EQE among all of the QDs, which is most likely attributed to their higher secondary absorption intensity. To observe the actual photon-to-electron process generated by the QDs in this system, the near-IR EQE data were divided according to their corresponding absorption to extract the value of the absorbed photon-to-current efficiency (APCE) ${ }^{15}$ based on the following equation:

$$
\mathrm{APCE}=\frac{E Q E}{1-10^{-a b s}}
$$

In Figure 5d, the APCE values are plotted versus the ratio between the photon energy $\left(\mathrm{E}_{h v}\right)$ and the QD band gap $\left(\mathrm{E}_{\mathrm{g}}\right)$. Overall, six critical peaks $\left(\mathrm{E}_{h v} / \mathrm{E}_{\mathrm{g}}=0.41,0.44,0.49,0.53,0.55\right.$ and 0.58$)$ were obtained; three of these peaks match well with theoretical simulation $^{16}$. Only the cells containing G-QDs and Y-QDs have maximum APCE values exceeding 100\%; the maximum was observed in the device with Y-QDs $(200 \%)$ at $0.49\left(\sim 1 / 2 \mathrm{E}_{\mathrm{g}}\right)$. This APCE indicates that two photons were simultaneously absorbed by the QDs and shows that the cell efficiency can also be enhanced by utilizing a nonlinear mechanism in DSSCs with non-concentrated light. The energy range of 1.24 to $1.12 \mathrm{eV}$ (lower than the QD bandgap of $\sim 2.64$ to $2.21 \mathrm{eV}$ ) is still energetically favorable for photon collection and current generation, although Ru-dye (N719) cannot absorb in this energy range. This feature offers a prospect in utilizing QDs as up-converters in DSSCs by transforming lower-energy photons to higher-energy photons.

Device optimization. To maximize cell performance, one strategy is to hybridize CdSe-PbSe QDs, where the calculated bandgaps of CdSe and $\mathrm{PbSe}$ are $2.37 \mathrm{eV}$, and $0.94 \mathrm{eV}$, respectively (Figure S12). The hybrid CdSe-PbSe QDs also demonstrate an evidence of broadband energy transfer based on a broad EQE enhancement in the UV-Vis range (Figure S13a). This enhancement implies that PbSe QDs and CdSe QDs could work as $p-n$ junctions when their energies are 

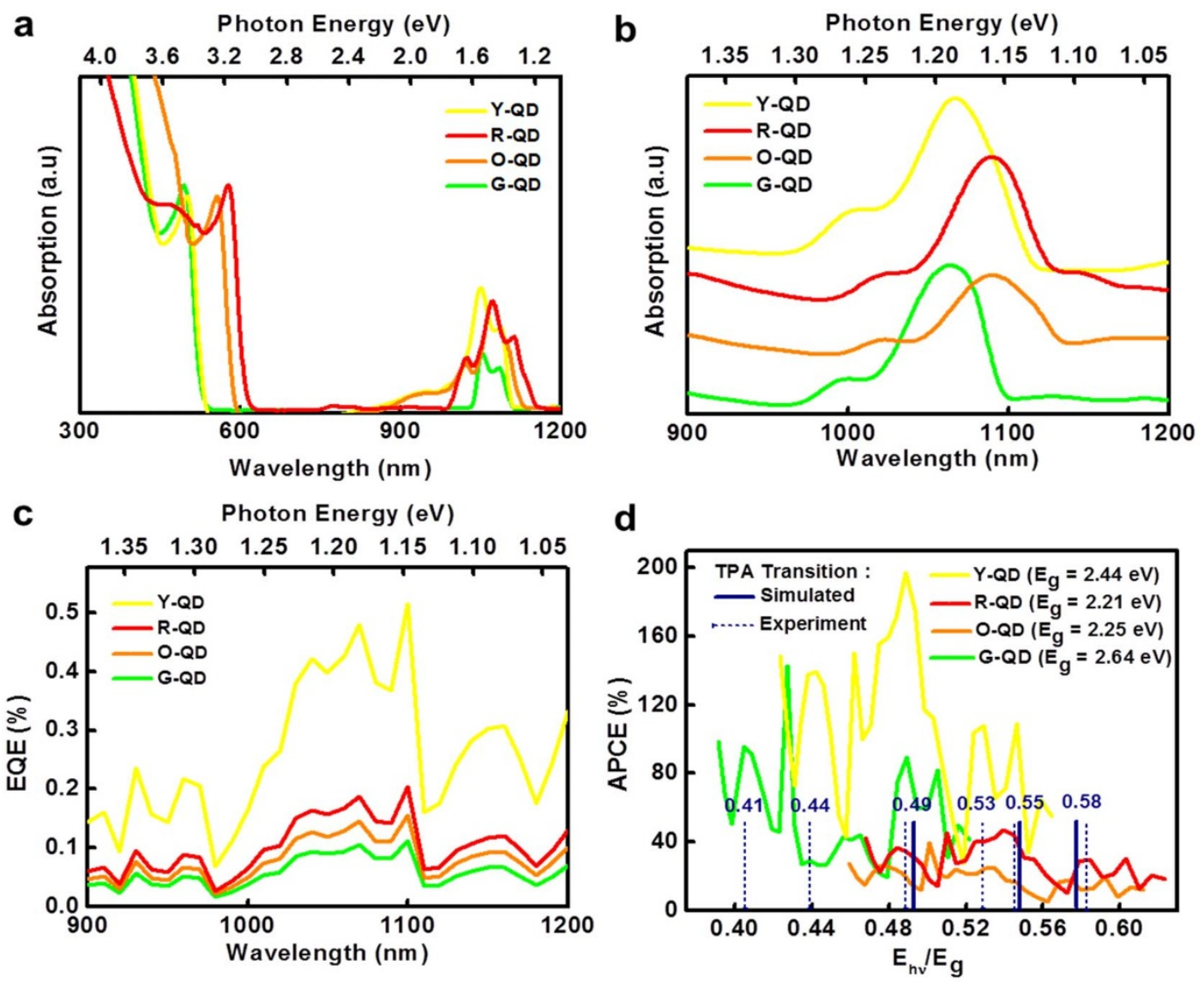

Figure 5 Two-photon absorption (TPA) and its photon-to-current efficiency. (a) Absorption of four different CdSe QDs in toluene with two distinct peaks in the visible range $(\sim 450 \mathrm{~nm}$ to $600 \mathrm{~nm})$ and near-IR range ( $\sim 900 \mathrm{~nm}$ to $1200 \mathrm{~nm})$. (b) Absorption of the CdSe QDs in ionic liquid at the near IR range. (c) The near-IR EQE of cells with QDs. (d) Absorbed photon-to-current efficiency (APCE) of cells with QDs versus $\hbar \omega / \mathrm{E}_{\mathrm{g}}$ (the incoming photon energy divided by each QD band gap) showing broad spectral spreading in which the TPA transition is represented by six peaks (dashed blue lines), three of which match well with theoretical simulation ${ }^{[16]}$ (solid blue lines). The thickness of the $\mathrm{TiO}_{2}$ photo-electrode is $\sim 8 \mu \mathrm{m}$, and the data were measured under the AM 1.5 G standard.

aligned $^{17,18}$. The PCE value obtained using this hybridization in an ionic liquid electrolyte is $6.04 \%$ (Figure S13b), while the PCE is $4.94 \%$ for the single PbSe QDs. The PCE enhancement is dependent on the $J_{s c}$ and $V_{o c}$ values. The lower $V_{o c}$ values for the PbSe QDs might be due to a downward shift of the $\mathrm{TiO}_{2}$ conduction band edge relative to the electrolyte containing PbSe QDs, and an upward shift may occur for the CdSe QDs, such that the cell on CdSe QDs has a higher $V_{o c}$ than that of the PbSe QDs. In addition, we find that the cosensitization of dyes and PbS QDs to mobile CdSe QDs in an acetonitrile-based volatile electrolyte also boost the efficiency to 8.83\% (Supplementary Information Figure S13 and Table S2) compared to $6.76 \%$ for the reference cell. These enhancements in non-volatile and volatile electrolytes demonstrate that mobile semiconductor quantum dots in electrolyte are very effective for boosting solar cell efficiency without any additional plasmonic or scattering layer, which has thus far limited the fabrication of thin and transparent solar cells.

\section{Discussion}

This report has demonstrated that the performance of dye-sensitized solar cells can be dramatically increased by using quantum dots (i.e. $\mathrm{CdSe}$, and hybrid $\mathrm{CdSe}-\mathrm{PbSe}$ ) dispersed in ionic liquid electrolyte. Two sets of data have been provided to support the role of QDs: 1) optical spectroscopy data showing that CdSe QDs serve as effective photon mediators for the dye and significantly increase the broad absorption of the sensitizing dye over the entire UV-Vis (300$800 \mathrm{~nm}$ ) and in part of the near IR range (900-1200 nm); and 2) external quantum efficiency (EQE) measurements showing that QDs markedly improve the photon-to-current efficiency via broadband energy transfer mediated by the QDs to the dye. This broadband energy transfer was demonstrated based on linear and quadratic mechanisms. The linear process was based on Föster resonance energy transfer (FRET) and co-sensitization of quantum dots, which facilitates the efficient reduction of oxidized dyes. The quadratic process was analyzed by two-photon absorption (TPA). Moreover, the sequential trend of each contribution by the QDs was found to vary in each mechanism.

\section{Methods}

Synthesis of functionalized quantum dots. All chemicals were purchased from Sigma Aldrich unless otherwise specified. CdSe quantum dots were synthesized using the organo-metallic synthesis method in the presence of amine organic coordinating ligands ${ }^{1,20}$. The growth rate of the QDs was controlled by the reaction times and temperatures which determine the bandgap. Toluene and hexane (Duksan Chemical) were sequentially used to dilute and store the initial QDs. QDs capped with thiol ligands were prepared as follows: First, $18 \mathrm{~mL}$ of original amino QDs in toluene was mixed with $9 \mathrm{~mL}$ of a $0.5-0.1 \mathrm{M}$ methanol solution of 2-dimethylaminoethane-thiol hydrochloride (DAET). The mixture was then stirred overnight at room temperature with argon under dark conditions until slight flocculation was observed, and a total of $3 \mathrm{~mL}$ of distilled water was sequentially added every 20-40 minutes. Separation of the system into two parts (toluene above water), in which QDs were transferred into the water phase, was observed after stirring for 3-4 hours. Un-reacted chemicals were removed by decanting and by a rotary evaporator at aspirator pressures. To extract the QDs from the water, $9 \mathrm{~mL}$ of ionic liquid, 1-ethyl-3-methylimidazolium bis (trifluoromethylsulfonyl) imide (EMIm-TFSI) was added and then was vigorously shaken until all of the QDs were transferred into the ionic liquid below the water phase. Any remaining traces were removed until the mixture was visibly clear. The 
synthesis of PbSe-QDs ${ }^{21}$ typically involve the reaction of as-synthesized oleic acid(OLA-) capped PbSe-QDs with 1-octadecene as a reaction medium with dithiol sodium (DT) at a relevant concentration with respect to the OLA (5:1 in our work). The functionalization sequence of the CdSe QDs was also applied to the PbSe QDs.

Device fabrication. FTO glass (TEC15, $15 \Omega / \mathrm{sq}$, Pelkington, USA), $1.5 \times 2 \mathrm{~cm}$, was sequentially cleaned with triton-X, acetone, and ethanol in an ultra-sonication bath, each for 20 minutes. The clean FTO was dried under a nitrogen gas flow before $\mathrm{TiCl}_{4}$ treatment. The $\mathrm{TiCl}_{4}$ treatment was conducted by immersing the clean FTOs into $40 \mathrm{mM} \mathrm{TiCl}$ solution at $80^{\circ} \mathrm{C}$ for $30 \mathrm{~min}$ and then rinsing with water and ethanol. The photo-electrode pastes (Ti-Nanoxide, Solaronix) were then doctor bladed onto the FTO substrate, followed by gradual sintering steps at $150^{\circ} \mathrm{C}(20 \mathrm{~min}), 350^{\circ} \mathrm{C}$ $(15 \mathrm{~min})$, and $500^{\circ} \mathrm{C}(30 \mathrm{~min})$. The surface of the photo-electrode $(\mathrm{PE})$ was cleaned using UV ozone treatment ( $30 \mathrm{~min}$ ) before being immersing the PE into a $0.3 \mathrm{mM}$ acetonitrile/tert-butanol $(1: 1)$ solution of N719 dye for 20 hrs at $2 \sim 8^{\circ} \mathrm{C}$. The counter-electrodes (CEs) were prepared by dip-coating of drilled-clean FTO in $0.06 \mathrm{mM}$ chloroplatinic acid hexahydrate $\left(\mathrm{H}_{2} \mathrm{PtCl}_{6} \cdot 6 \mathrm{H}_{2} \mathrm{O}\right)$ in ethanol solution followed by sintering at $380^{\circ} \mathrm{C}(20 \mathrm{~min})$. The $\mathrm{PE}$ and $\mathrm{CE}$ were sandwiched with Surlyn film $\left(60 \mu \mathrm{m}\right.$, Solaronix) used as a sealing agent at $80^{\circ} \mathrm{C}$. The electrolyte based on EMIm-TFSI ionic liquid consisting of EMIm-I $(0.5 \mathrm{M})$, tBP $(0.36 \mathrm{M})$, I $(0.13 \mathrm{M})$, and $\mathrm{I}_{3}{ }^{-}(0.01 \mathrm{M})$ was injected into the cell through the holes drilled on the CE.

Optical characterization. The absorption spectra were obtained using UV-Vis-NIR spectrophotometers (UV-3600, Shimadzu) with a $150 \mathrm{~mm}$ integrating sphere. The emission spectra were recorded using a PL system composed of a monochromator (0.1-0.3 nm triple grating, Acton series spectrograph (ARC) 300i spectrometer, NCL controller, Princeton Instrument), a 532-nm solid state laser diode (Spectra Physics) light source with high power $(40 \mathrm{~mW})$, and a detector (MCP-PMT, Hamamatsu). The absorption time decay was measured using nanosecond time-resolved absorption spectrum analysis (C7700 Hamamatsu) with a nanosecond Q-SW YAG laser $\left(\lambda=532 \mathrm{~nm}, 30 \mathrm{~Hz}\right.$ repetition rate, and $23-50 \mu \mathrm{J} \mathrm{cm}^{-1} /$ pulse). The emission time decay was determined from time-resolved PL decay measurements performed using a PL 920 emission lifetime apparatus (Edinburgh Instrument), with a 100-ps to $50-\mu$ s lifetime range (TCSPC), an excitation range of $375 \mathrm{~nm}$ to $470 \mathrm{~nm}$, and a repetition-rate range of $10 \mathrm{kHz}$ to $100 \mathrm{MHz}$.

Photovoltaic characterization. Current density-voltage $(J-V)$ data were measured under simulated AM $1.5 \mathrm{G}$ illumination at $100 \mathrm{~mW} / \mathrm{cm}^{2}$ (PEC-L 11, Peccell Technologies) with less than a $25 \%$ spectral mismatch and a thermostat controller (set to $23 \sim 26^{\circ} \mathrm{C}$ ). The active area of each cell was carefully determined with a digital microscope $\left(0.16 \pm 0.02 \mathrm{~cm}^{2}\right)$. EQE spectra were recorded using a spectrum measurement system (PEC-S2026, Bunkoukeiki Co. Ltd.), composed of a Xenon lamp light source $(150 \mathrm{~W})$, a monochromator with a 600 -grooves $/ \mathrm{mm}$ grating, and a $0.1 \mathrm{~mm}$ slit that provides $\pm 2 \mathrm{~nm}$ or less wavelength accuracy. The EQE measurement was screened in two steps: in the UV-Vis range of 300-800 nm and then in the near-IR range of $900-1200 \mathrm{~nm}$ by different calibrations. The absolute EQE values in the near-IR range were validated by: 1) comparing the data with the $\mathrm{EQE}$ of cells without QDs as a baseline; 2) correcting the data with the maximum attainable value from noise $(<0.01 \%) ; 3)$ verifying the accuracy using two different calibrated photodiode standards (Vis-near IR Si and near IR-IR Ge); and 4) using a five-time shatter resistant filter, which was calibrated with the SiPD spectra sensitivity to detect a small photocurrent signal. All of the representative data were collected from 10-15 measured cells for each treated condition with $86 \%$ accuracy.

1. Han, S.-H., Lee, S., Shin, H. \& Jung, H. S. A quasi-inverse opal layer based on highly crystalline $\mathrm{TiO}_{2}$ nanoparticles: a new light-scattering layer in dyesensitized solar cells. Advanced Energy Materials. 1, 546-550 (2011).

2. Qi, J., Dang, X., Hammond, P. T. \& Belcher, A. M. Highly efficient plasmonenhanced dye-sensitized solar cells through metal@oxide core-shell nanostructure. ACS Nano. 9, 7108-7116 (2011).

3. Kuang, D. et al. Co-sensitization of organic dyes for efficient ionic liquid electrolyte-based dye sensitized solar cells. Langmuir. 23, 10906-10909 (2007)

4. Hardin, B. E. et al. Increased light harvesting in dye sensitized solar cells with energy relay dyes. Nature Photonics. 3, 406-411 (2009).

5. Yang, J., Sargent, E. H., Kelley, S. O. \& Ying, J. Y. A general phase transfer protocol for metal ions and its application in nanocrystal synthesis. Nature Materials. 8, 683-689 (2010).

6. Guyot-Sionnest, P. Charging colloidal quantum dots by electrochemistry. Microchim Acta. 160, 309-314 (2008).
7. Halpert, J. E. et al. Electrostatic formation of quantum dot/J-aggregate FRET pairs in solution. J.Phys.Chem.C. 113, 9986-9992 (2009).

8. Jeon, J. et al. The role of confined water in ionic liquid electrolytes for dye sensitized solar cells. J.Phys.Chem.Lett. 3, 556-559 (2012).

9. Lunz, M. et al. Concentration dependence of Föster resonance energy transfer between donor and acceptor nanocrystal quantum dot layers: effect of donordonor interaction. Phys. Rev. B. 83, 115423 (2011).

10. Luther, J. M., Jain, P. K., Ewers, T. \& Alivisatos, A. P. Localized surface plasmon resonance arising from free carriers in doped quanutm dots. Nature Materials. 10, 361-366 (2011).

11. Ito, S. et al. Bifacial dye-sensitized solar cells based on an ionic liquid electrolyte. Nature Photonics. 2, 693-698 (2008).

12. Shalom, M., Rühle, S., Hod, I., Yahav, S. \& Zaban, A. Energy level alignment in CdSe quantum dot sensitized solar cells using molecular dipoles. J. Am. Chem. Soc. 131, 9876-9877 (2009).

13. Yaacobi-Gross, N. et al. Molecular control of quantum-dot internal electric field and its application to CdSe-based solar cells. Nature Materials. 10, 974-979 (2011).

14. He, G. S. et al. Multi-photon excitation properties of CdSe quantum dots solutions and optical limiting behavior in infrared range. Optics Express. 15, 12818-12833 (2007).

15. Semonin, O. E. et al. Peak external photocurrent quantum efficiency exceeding $100 \%$ via MEG in a quantum dot solar cell. Science. 334, 1530-1533 (2011).

16. Padilha, L. A. et al. Optimization of band structure and quantum-size-effect tuning for two-photon absorption enhancement in quantum dots. Nano Letter. 11, 1227-1231 (2011)

17. Tachan, Z. et al. $\mathrm{PbS}$ as a Highly catalytic counter electrode for polysulfide-based quantum qot solar cells. J.Phys.Chem.C. 5, 6162-6166 (2011).

18. Pattantyus-Abraham, A. G. et al. Depleted-heterojunction colloidal quantum dot solar cells. ACS Nano. 4, 3374-3380 (2010).

19. Talapin, D. V., Rogach, A. L., Kornowski, A., Haase, M. \& Weller, H. Highly luminescent monodispersed $\mathrm{CdSe}$ and $\mathrm{CdSe} / \mathrm{ZnS}$ nanocrystals synthesized in a hexadecylamin-trioctylphosphineoxide-trioctylphosphine mixture. Nano Letter. 1, 207-211 (2001).

20. Wuister, S. F., Donegá, C.d. M. \& Meijerink, A. Influence of thiol capping on the exciton luminescence and decay kinetics of $\mathrm{CdTe}$ and $\mathrm{CdSe}$ quantum dots. J.Phys.Chem.B. 108, 17393-17397 (2004).

21. Moody, I. S., Stonas, A. R. \& Lonergan, M. C. PbS nanocrystals functionalized with a short-chain, ionic, dithiol ligand. J.Phys.Chem.C. 112, 19383-19389 (2008).

\section{Acknowledgments}

This research was supported by the Korea Center for Artificial Photosynthesis (NRF-2011C1AAA001-2011-0030280) and the Hybrid Interface Materials R\&D Center (HIMC) of Global Frontier Project funded by the Ministry of Science, ICT \& Future Planning. Also, it was supported by the National Research Foundation grants (2011-0028737, 2010-0029042, 2012-0001174), by Priority Research Centers Program (2011-0031407), and by the WCU program (R-31-2008-000-10055-0). The authors would like to acknowledge the assistance of Dr. N.Tsvetkov and Prof. A.B. Tae for cross-checking the near-IR EQE data and Dr. I. Usman for the guidance regarding optical spectroscopy characterization.

\section{Author contributions}

G.W.P.A., G.I.L., and J.K.K. initially proposed the main idea. Material synthesis, optical characterisation, device fabrication, and data analysis are mainly conducted by G.W.P.A. and G.I.L. Comparison of plasmonic framework and calculation have been performed by S.-W.B and J.-Y. Lee. All the results and mechanisms were discussed by all authors.

\section{Additional information}

Supplementary information accompanies this paper at http://www.nature.com/ scientificreports

Competing financial interests: The authors declare no competing financial interests.

How to cite this article: Adhyaksa, G.W.P., Lee, G.I., Baek, S., Lee, J. \& Kang, J.K. Broadband energy transfer to sensitizing dyes by mobile quantum dot mediators in solar cells. Sci. Rep. 3, 2711; DOI:10.1038/srep02711 (2013).

This work is licensed under a Creative Commons AttributionNonCommercial-NoDerivs 3.0 Unported license. To view a copy of this license, visit http://creativecommons.org/licenses/by-nc-nd/3.0 